\title{
Regulation of secondary metabolism in Vitis vinifera by an extract of A.nosodum effects of physiology and grape quality.
}

\author{
Linda Salvi ${ }^{1 *}$, Cecilia Brunetti $^{1}$ and Giovan Battista Mattii ${ }^{1}$ \\ ${ }^{1}$ DISPAA, Dipartimento di Scienze delle Produzioni Agroalimentari e dell'Ambiente, Università di Firenze, Viale delle Idee 30, \\ 50019, Sesto Fiorentino (FI), Italia
}

\begin{abstract}
Heat and drought are major abiotic stresses that impact on many Vitis vinifera physiological processes and berry development, especially as global climate change is speeding up the expected frequency and severity of both stress factors. Nevertheless, seaweed extracts might represent a strategy to confer to treated plants the capability to be more prone to counteract shortterm periods of severe stresses. In this paper, we report the effects of foliar treatments with an Ascophyllum nodosum extract on physiological and biochemical parameters of grapevine. In our study performed in the 2016 season in open field in Tuscany, we assessed differences in leaf gas exchanges, chlorophyll fluorescence and midday stem water potential between treated and untreated vines. In addition, the in-depth analysis of anthocyanins and flavonols in berries presented within the work adds significantly to our knowledge of the influence of seaweed extract on the phenylpropanoid pathway. Moreover, our work witness the treatment-promoting greater tolerance to stress in vines, and support previous studies that hypothesize an influence of treatments with algae extracts on qualitative characteristics in V.vinifera.
\end{abstract}

\section{Introduzione}

Il clima dell'areale viticolo toscano è caratterizzato da estati con elevate temperature e scarsa piovosità. Queste condizioni influenzano lo stato fisiologico della vite e accelerano i processi di maturazione delle bacche, che a volte possono determinare vini sbilanciati, con alto contenuto alcolico e basse concentrazioni polifenoliche [1].

Negli ultimi anni, i ricercatori hanno studiato il ruolo dei biostimolanti, e tra essi degli estratti di alghe, come possibili promotori dell'acclimatazione delle piante nel breve termine [2]. A questo proposito, gli estratti di alghe sembrano essere responsabili di una maggiore biosintesi dei metaboliti secondari antiossidanti, ad esempio di polifenoli [3], che sono coinvolti sia nella risposta agli stress abiotici, che nella qualità dell'uva.

Nonostante il numero crescente di articoli scientifici sull'effetto dei trattamenti con estratti di alghe sulle prestazioni delle colture in condizioni di stress abiotico, sull'efficienza nell'uso dell'acqua e l'attivazione delle vie biosintetiche di composti antiossidanti [4], molti di questi prodotti non sono stati pienamente sfruttati a causa della mancanza di dati scientifici sulle molecole bioattive presenti nelle alghe e sul loro modo di influenzare le performances delle piante. Fino ad ora, molti ricercatori hanno analizzato il potenziale "biostimolatore" degli estratti di alghe concentrandosi separatamente sugli scambi gassosi o sul contenuto fenolico [5-9]. Con questo progetto ci si propone di esaminare contemporaneamente la risposta ecofisiologica della pianta, $i$ fattori di qualità dell'uva e i composti antiossidanti negli acini, al fine di ottenere una visione integrata degli effetti del trattamento.

L'obiettivo principale di questo studio è stato quello di valutare l'impatto di un estratto alcalino di Ascophyllum. nodosum sull'attività fisiologica, sulla resa e sui parametri qualitativi delle uve (Vitis vinifera, cv. Sangiovese), rivolgendo particolare interesse al raggiungimento dell'equilibrio tra maturità tecnologica $\mathrm{e}$ fenolica. L'ipotesi di ricerca è che l'applicazione di questi estratti, grazie ai suoi composti bioattivi, potrebbe aumentare sia la tolleranza alla siccità che la qualità dell'uva innescando la biosintesi di metaboliti secondari.

\section{Materiali e metodi}

La ricerca è stata condotta durante la stagione vegetativa 2016 presso l'azienda agricola Villa Montepaldi, che si trova sulle colline di San Casciano Val di Pesa (FI), nella parte settentrionale della nota zona di produzione del Chianti Classico. Il vigneto nel quale è stata effettuata la sperimentazione è stato impiantato nel 1998 con viti di Sangiovese (clone R 24) innestate su 420A, esposto a Sud-Ovest, con sesto d'impianto di 2,8 x 0,9 e forma d'allevamento a controspalliera, potata a cordone speronato. Il suolo è di medio impasto, con tessitura

\footnotetext{
Corresponding author: linda.salvi@unifi.it
} 
franco-argillosa (argilla 38.8\%; sabbia 23.4\%; limo $37.8 \%$ ), con $\mathrm{pH}$ leggermente alcalino (7.8). Avvalendosi del disegno sperimentale a blocchi randomizzati con 5 ripetizioni per tesi di 3 filari ciascuna, è stato impostato il confronto tra il non trattato (CTRL) e il trattatoa con un estratto di $A$. nodosum (SWE), distribuito per via fogliare con dose di 4 1/ha, ad inizio invaiatura e due settimane dopo. Negli stessi giorni dei trattamenti con $A$. nodosum, le viti non trattate sono state spruzzate con acqua. Dal filare centrale di ogni ripetizione, sono state selezionate e cartellinate 15 viti omogenee/tesi, usate per i rilievi eco-fisiologici, produttivi e per i campionamenti di acini, in tre fasi fenologiche: pre-invaiatura (pretrattamento), piena invaiatura (una settimana dopo il primo trattamento) e maturazione (una settimana dopo il secondo trattamento). Ad ogni rilievo, campionando 15 foglie adulte e sane poste nella fascia mediana della parete dalle piante cartellinate di ogni tesi, sono stati effettuati rilievi di fotosintesi $\left(\mathrm{P}_{\mathrm{n}}, \mu \mathrm{mol} \mathrm{m}^{-2} \mathrm{~s}^{-1}\right) \mathrm{e}$ conduttanza stomatica $\left(g_{s}, \mathrm{mmol} \mathrm{m}^{-2} \mathrm{~s}^{-1}\right)$ con un analizzatore di gas all'infrarosso (Ciras 3, PP Systems, USA), di efficienza massima del fotosistema II (Fv/Fm) con un fluorimetro portatile (Handy-PEA ${ }^{\circledR}$, Hansatech Instruments, UK), di potenziale idrico di tralcio $\left(\Psi_{\mathrm{m}}\right.$, $\mathrm{Mpa}$ ) con una camera a pressione (PMS Instrument Co, USA). Inoltre, nelle stesse tre fasi fenologiche, sono stati effettuati campionamenti di 150 acini da ogni ripetizione/tesi per la determinazione degli zuccheri solubili (g/L), dell'acidità titolabile $(\mathrm{g} / \mathrm{L}$ di acido tartarico), del $\mathrm{pH}$ e del peso dell'acino (g). Su un sottocampione analogo di 30 acini sono state effettuate le analisi relative alla composizione di antociani e polifenoli nelle bucce ( $\mu$ mol g di sostanza secca ${ }^{-1}$ ), utilizzando un HPLC-DAD (Perkin Elmer, USA).

Alla raccolta, sulle 10 piante cartellinate per ogni ripetizione di ogni tesi sono stati determinati il peso fresco della produzione $(\mathrm{Kg})$, il numero di grappoli/pianta e il peso fresco del grappolo $(\mathrm{g})$.

I dati raccolti sono stati sottoposti all'analisi della varianza ad una via con SPSS Data Editor (IBM, USA). Le differenze statisticamente significative sono state assunte per $\mathrm{P}<0.05$.

\section{Risultati e discussioni}

\subsection{Effetto dell'estratto di $A$. nodosum sui parametri eco-fisiologici}

In Tab. 1 sono riassunti i valori medi stagionali dei parametri eco-fisiologici misurati durante la stagione. SWE mostra valori medi di $\mathrm{P}_{\mathrm{n}}, \quad g s$ e Fv/Fm significativamente maggiori di CTRL. Al contrario, nel nostro studio, non abbiamo registrato differenze significative in $\Psi_{\mathrm{m}}$ tra SWE e CTRL. I trattamenti con $A$. nodosum hanno avuto un effetto significativo nel ridurre la chiusura stomatica e aumentare la fotosintesi. In più, mentre le piante non trattate hanno mostrato segni di moderata foto-inibizione, le viti trattate sono state in grado di mantenere i valori $\mathrm{Fv} / \mathrm{Fm}$ più vicini all'optimum indicato per la vite $(\sim 0,80)$ [10-11]. Gli effetti positivi sulle funzioni fisiologiche delle piante indotte dai trattamenti con $A$. nodosum sono stati precedentemente riportati anche in spinacio, Spiraea e Pittosporum [9; 12]. Ipotizziamo che il miglioramento delle prestazioni fisiologiche promosse dai trattamenti con A. nodosum possa essere correlato alla presenza di aminoacidi e composti fenolici all'interno dell'estratto, che, con probabilità, hanno conferito un'elevata capacità antiossidante all'estratto stesso.

Tab 1. Valori medi stagionali dei parametri eco-fisiologici.

\begin{tabular}{|c|c|c|}
\hline Parametro & SWE & CTRL \\
\hline $\boldsymbol{P}_{\boldsymbol{n}}\left(\boldsymbol{\mu} \mathbf{m o l ~ \mathbf { ~ m } ^ { - 2 } \mathbf { ~ s } ^ { - 1 } )}\right.$ & $11.1 \pm 0.8 \mathrm{a}$ & $9.3 \pm 0.6 \mathrm{~b}$ \\
\hline $\boldsymbol{g s}\left(\mathbf{m m o l ~ \mathbf { m } ^ { - 2 } \mathbf { s } ^ { - 1 } )}\right.$ & $245 \pm 16.5 \mathrm{a}$ & $197 \pm 16 \mathrm{~b}$ \\
\hline $\mathbf{F v} / \mathbf{F m}$ & $0.79 \pm 0.01 \mathrm{a}$ & $0.76 \pm 0.01 \mathrm{~b}$ \\
\hline $\boldsymbol{\Psi}_{\mathbf{m}}(\mathbf{M p a})$ & $-1.13 \pm 0.1 \mathrm{a}$ & $-1.23 \pm 0.07 \mathrm{a}$ \\
\hline
\end{tabular}

\subsection{Effetto dell'estratto di $A$. nodosum sulle caratteristiche qualitative e produttive in Vitis vinifera}

I valori a vendemmia degli zuccheri totali e dei parametri produttivi sono presentati nella Tab. 2. Non sono state registrate differenze statistiche tra $\mathrm{i}$ due trattamenti nell'acidità totale e nel pH (dati non mostrati) e nei parametri produttivi. Al contrario, differenze significative nel tenore di zucchero totale sono state osservate a vendemmia tra acini SWE e CTRL. A piena maturazione, SWE presentava un contenuto di zuccheri totali inferiore di circa $10 \mathrm{~g} / \mathrm{L}$ rispetto a CTRL.

Tab 2. Zuccheri solubili e parametri produttivi a vendemmia.

\begin{tabular}{|c|c|c|}
\hline Parametro & SWE & CTRL \\
\hline Zuccheri (g/L) & $229 \pm 1 \mathrm{~b}$ & $239 \pm 1 \mathrm{a}$ \\
\hline Peso di 150 acini (g) & $375 \pm 15 \mathrm{a}$ & $360 \pm 15 \mathrm{a}$ \\
\hline Peso di 10 grappoli (Kg) & $3.8 \pm 0.1 \mathrm{a}$ & $3.5 \pm 0.3 \mathrm{a}$ \\
\hline Resa di 10 piante (Kg) & $27 \pm 2 \mathrm{a}$ & $26 \pm 3 \mathrm{a}$ \\
\hline
\end{tabular}

Per quanto riguarda la composizione delle bucce, sono stati quantificati i maggiori contenuti di antociani sia metossilati (peonidina, petunidina, malvidina) che non metossilati (delfinidina e cianidina) (Fig. 1 e Fig. 2), a piena invaiatura e a maturazione. Il rapporto tra i derivati della quercetina e del kampferolo è anche esso maggiore 
nelle bucce SWE rispetto a CTRL, in tutte le fasi fenologiche post trattamento (Fig. 3).

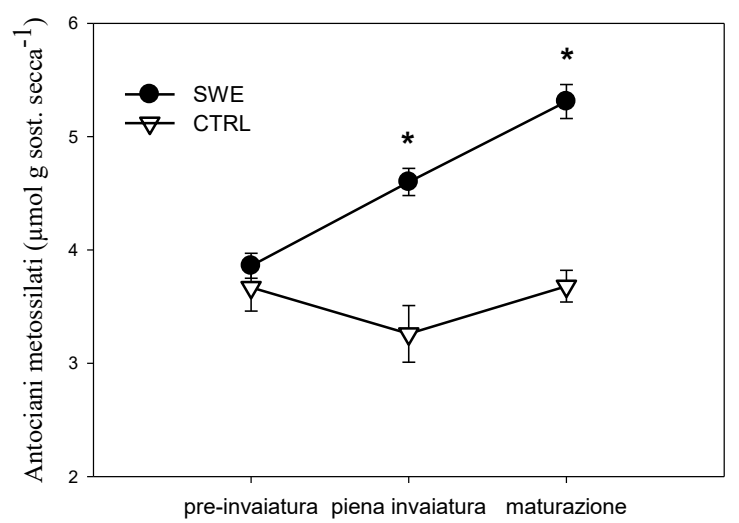

Fig 1. Valori medi $( \pm \mathrm{SE}, \mathrm{n}=5)$ di antociani metossilati nelle bucce, nei tre punti di rilievo. * indica significatività $(\mathrm{P} \leq 0.05)$

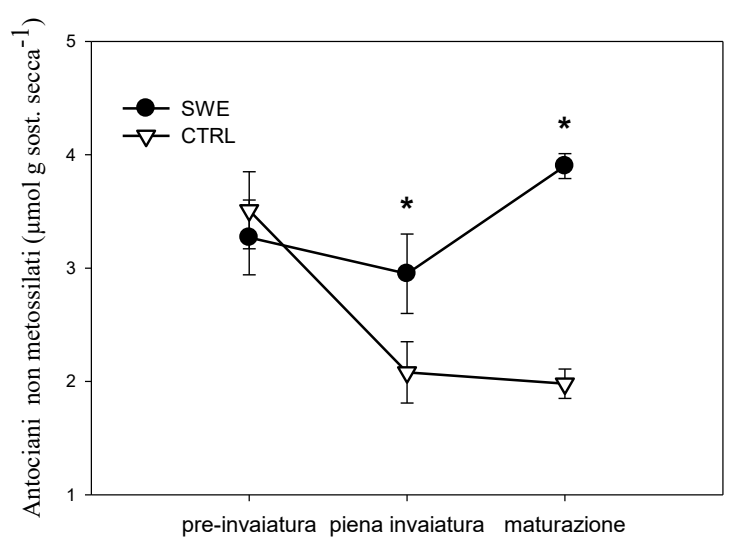

Fig 2. Valori medi $( \pm \mathrm{SE}, \mathrm{n}=5)$ di antociani non metossilati nelle bucce, nei tre punti di rilievo. * indica significatività $(\mathrm{P} \leq$ $0.05)$

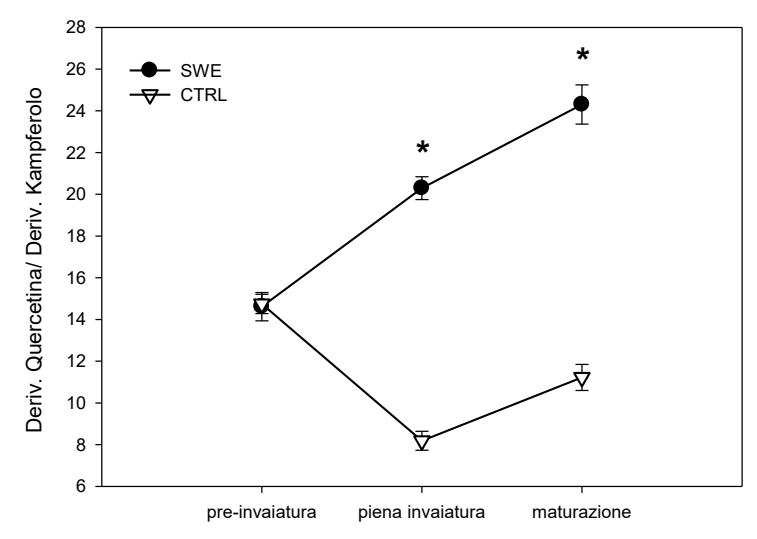

Fig 3. Valori medi ( \pm SE, $n=5$ ) del rapporto tra derivati della quercetina e derivati del kampferolo nelle bucce, nei tre punti di rilievo. * indica significatività $(\mathrm{P} \leq 0.05)$
Analizzando collettivamente questi risultati, possiamo ipotizzare che le elevate temperature stagionali abbiano guidato l'accelerazione dell'accumulo di zucchero in CTRL. Questo effetto è meno evidente in SWE, in cui abbiamo osservato un contenuto zuccherino significativamente inferiore accompagnato da un più elevato contenuto di antociani rispetto alle piante CTRL. Ciò è in accordo con studi precedenti [8;13] e sostiene fortemente l'ipotesi che i trattamenti con $A$. nodosum possano essere coinvolti nel ritardare la maturazione, bilanciando la maturazione tecnologica con quella fenolica. Tuttavia, questi effetti positivi sulla qualità dell'uva non sono stati accompagnati da un concomitante incremento della resa, probabilmente perché l'estratto di $A$. nodosum è stato distribuito in stagione avanzata su acini completamente sviluppati in dimensioni. Al contrario, come riportato in precedenza nell'uva da tavola, applicazioni di biostimolanti durante la divisione e distensione delle cellule dell'acino potrebbero persino indurre un miglioramento della produttività della vite e del peso delle bacche [14-16].

\section{Conclusioni}

Nell'ottica di ridurre i fenomeni di sfasamento tra maturità tecnologica e fenolica ascrivibili alle interazioni tra i cambiamenti climatici in atto ed alle mutate tecniche di gestione della chioma, la distribuzione fogliare di prodotti biostimolanti può rappresentare una valida pratica agronomica.

I nostri dati indicano che i trattamenti con $A$. nodosum durante le ultime fasi dello sviluppo delle bacche possono potenziare le prestazioni di $V$. vinifera migliorando i parametri eco-fisiologici e influenzando il metabolismo secondario, con conseguente miglioramento della qualità dell'uva.

In dettaglio, i trattamenti con l'estratto di $A$. nodosum hanno aumentato la fotosintesi, riducendo la chiusura stomatica, massimizzato l'efficienza del fotosistema II e bilanciato la maturazione dell'uva. Questo prodotto risulta essere un valido aiuto in annate siccitose e calde, permettendo di ovviare al problema di un eccesso di zucchero nell'acino a vendemmia, riuscendo a ritardare la maturità tecnologica della bacca e apportando un corredo polifenolico più alto.

Al fine di comprendere i meccanismi di azione degli estratti di alghe marine, è fondamentale concentrare la ricerca futura sull'identificazione dei composti chimici delle alghe con un potenziale effetto sulla via dei fenilpropanoidi delle piante trattate.

\section{Bibliografia}

1. J.R. Mosedale, K.E. Abernethy, R.E. Smart, R.J. Wilson, I. Maclean, Glob. Chang. Biol. 22, 3814-3828 (2016)

2. G.C. Popescu, M. Popescu, Current Trend in Natural Science 3(6), 61-67 (2014).

3. R.G. Gurav, J.P. Jadhav, Environ. Sci. Pollut. Res. 20, 4532-4539 (2013).

4. A. Santaniello, A. Scartazza, F. Gresta, E. Loreti, A. Biasone, D. Di Tommaso, A. 
Piaggesi, P. Perata, Front. Plant Sci. 8, 1362 (2017).

5. D. Fan, D.M. Hodges, J. Zhang, C.W. Kirby, X. Ji, S. J. Locke, A.T. Critchley, B. Prithiviraj, Food Chem. 124, 195-202 (2011).

6. N. Paradikovic, T. Vinkovic, I. Vrcek, I. Zuntar, M. Bojic, M. Medic-Saric, Plants. J. Sci. Food. Agric. 91, 2146-2152 (2011).

7. T.M. Spann, H.A. Little, Hort. Sci. 46(4), 577$582(2011)$.

8. A. Sabir, K. Yazar, F. Sabir, Z. Kara, M.A. Yazici, N. Goksu, Sci. Hortic. 175, 1-8 (2014).

9. C. Xu, D.I. Leskovar, Sci. Hort. 183, 39-47 (2015).

10. M. Bertamini, N. Nedunchezhian, Plant Sci. 164, 635-644 (2003).

11. E.H. Murchie, T. Lawson, J. Exp. Bot. 64 (13), 3983-3998 (2013).

12. H.O. Elansary, K. Skalicka-Woźniak, I.W. King, Plant Physiol. Bioch. 105, 310-320 (2016).

13. T. Frioni, P. Sabbatini, S. Tombesi, J. Norrie, S. Poni, M. Gatti, A. Palliotti, Sci. Hort. 232, $97-$ 106 (2018).

14. J. Norrie, T. Branson, P.E. Keathley, Acta Hortic. 594, 315-319 (2002).

15. J. Norrie, J.P. Keathley, Acta Hort. 727, 243247 (2006).

16. A.S. Khan, B. Ahmad, M.J. Jaskani, R. Ahmad, A.U. Malik, Int. J. Agric. Biol. 14, 383-388 (2012). 